\title{
Electrical resistance of carbon nanotube yarns under compressive transverse pressure
}

\author{
Toan Dinh, Tuan-Khoa Nguyen, Hoang-Phuong Phan, Jarred Fastier-Wooller, Canh-Dung Tran, Nam-Trung \\ Nguyen, Dzung Viet Dao,
}

\begin{abstract}
This paper reports on the impact of compressive pressure on the electrical properties of carbon nanotube (CNT) yarns fabricated by dry web-spinning and heat-treatment processes. Under increasing applied compressive pressure in the radial direction of the yarn, the electrical resistance of CNT yarns gradually decreased by $\mathbf{2 . 8} \%$ at a threshold applied pressure of $60 \mathrm{kPa}$ where the resistance change is saturated. The decrease of CNT resistance with increasing pressure is attributed to the increase in the volume fraction of CNT, resulting in the increase of the effective junctions between adjacent CNTs, and the reduction of the tunneling distance between single CNTs. CNT yarns embedded in elastomers show high potential as an advanced functional element for a wide range of mechanical sensing applications including flexible pressure and tactile sensing.
\end{abstract}

Index Terms-Carbon nanotube, pressure effect, yarn/fibre, volume fraction, tunneling resistance.

\section{INTRODUCTION}

$\mathbf{S}$ INCE its discovery in 1991, carbon nanotubes (CNTs) have been considered as an unprecedented material for mechanical sensing applications owing to their lightweight, superior mechanical strength and high conductivity [1], [2]. At the initial stage, the research on CNT materials has driven towards the investigation of fundamental mechanical and sensing properties of individual CNTs for extreme high performance sensors [3], [4], [5]. However, difficulties in the fabrication and implementation of single CNTs for practical applications are the main drawback which prevents the commercialisation of such single CNT sensors.

Recent research has focused on investigating the CNTbased macrostructures such as CNT membranes, CNT composites, and fibres for emerging applications including flexible/stretchable strain sensors, actuating devices, and energy harvesters. For instance, CNT films can be employed to develop MEMS (Micro Electro-Mechanical System) strain sensors [6], flexible structures for MEMS actuators [7], and thermoelectric portable devices [8]. However, these macro CNT structures do not inherit the unique mechanical and electrical properties of single CNTs due to the inhomogeneous distribution of CNTs in macrostructures. Therefore, a number of studies have been carried out to enhance mechanical sensing properties of CNT fibre-liked devices for sensing applications including embedded structural health monitoring (SHM) systems [5], [6], [7], [8], [9].

T. Dinh, T.K. Nguyen, H.P. Phan, J. Fastier-Wooller, N.-T. Nguyen, D. V. Dao, are with the Queensland Micro-Nanotechnology Centre, Griffith University, Queensland, Australia (e-mail: toan.dinh@griffithuni.edu.au).

C.-D. Tran is with School of Mechanical and Electrical Engineering, University of Southern Queensland, Queensland, Australia.

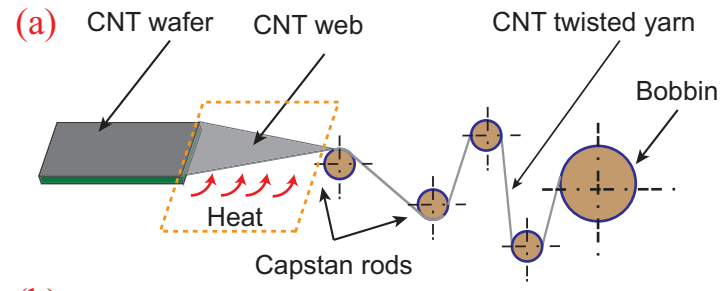

(b)

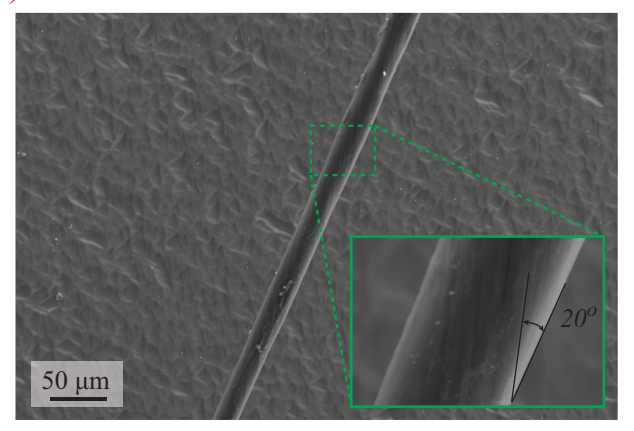

Fig. 1. Fabrication of CNT yarns. (a) Dry spinning process of twisted CNT yarns from a CNT wafer/forest [11], [13]. (b) Scanning Electron Microscopy (SEM) image of the as-fabricated micro-yarn. The inset shows the close-up SEM image of $12 \mu \mathrm{m}$-diameter CNT micro-yarn with a twisting angle of $20^{\circ}$.

CNT yarns are of high interest in mechanical sensing due to their high conductivity, high Young's modulus, low mass and capability of being embedded in other materials without modifying their structure. In order to achieve these potential characteristics, several spinning methods have been applied to increase the compactness of yarns, including dry spinning from CNT forests, aerogels, polymer solutions and films [10]. As a result, CNT yarns can achieve a Young modulus of $17 \mathrm{GPa}$, a tensile strength of $1.4 \mathrm{GPa}$, and conductivity of $3.1 \times 10^{5}$ $\mathrm{S} / \mathrm{m}$ [11]. To date, there have been a number of works on the strain sensitivity of CNT yarns, showing a relatively small gauge factor of lower than 0.5 [9], [12]. Furthermore, although many experimental studies on the effect of tensile stress on the electrical properties of CNT yarns have been reported, the electromechanical property of CNT yarns under compressive transverse pressure has not been fully understood.

By embedding CNT yarns inside elastomers, we investigated the electromechanical properties of CNTs yarns under radial pressure. Experimental results show that the resistance of our CNT yarns reduces to $2.8 \%$ with an applied pressure of $60 \mathrm{kPa}$, corresponding to a sensitivity of $4.7 \times 10^{-4} \mathrm{kPa}^{-1}$, which is comparable with highly sensitive MEMS pressure 
sensors. The pressure sensitive mechanism will be interpreted based on the tunneling conduction between individual CNTs.

\section{EXPERIMENTAL}

The growth of CNT forests on a silicon substrate was performed using a chemical vapor deposition (CVD) process. The CNT forests were then spun into yarns by a dry spinning process, as shown in Figure 1(a). CNT fibres were firstly pulled out from the wafer to form CNT web. The web was annealed in a furnace with adjustable temperature varying from 200 to $600^{\circ} \mathrm{C}$. This annealing process is to decrease the van der Waals interaction between adjacent CNTs, facilitating the smooth drafting of CNT fibres [1], [2], [11]. The alignment of CNTs can also be improved under the application of tension forces from the twisting system. Both tension and torque were generated from a capstan rod system to control the radial pressure of the yarns via contact angles on the rods. The yarns were twisted with a factor of 15,000 turns per meter (TMP). Subsequently, yarns with a diameter of $12 \mu \mathrm{m}$ were formed (Figure 1b). The close-up SEM image (the inset) in Figure $1 \mathrm{~b}$ shows the twist angle of approximately $20^{\circ}$; hence the volume fraction of spaces between the carbon nanotubes (porosity) of the CNT yarn can be approximated to be 0.75 [11], [14]. The corresponding volume fraction of CNTs is estimated to be 0.25 without applying pressure. Tensile testing was performed to determine the Youngs modulus and tensile strength of the yarns, which are approximately $16 \mathrm{GPa}$ and 1 $\mathrm{GPa}$, respectively.
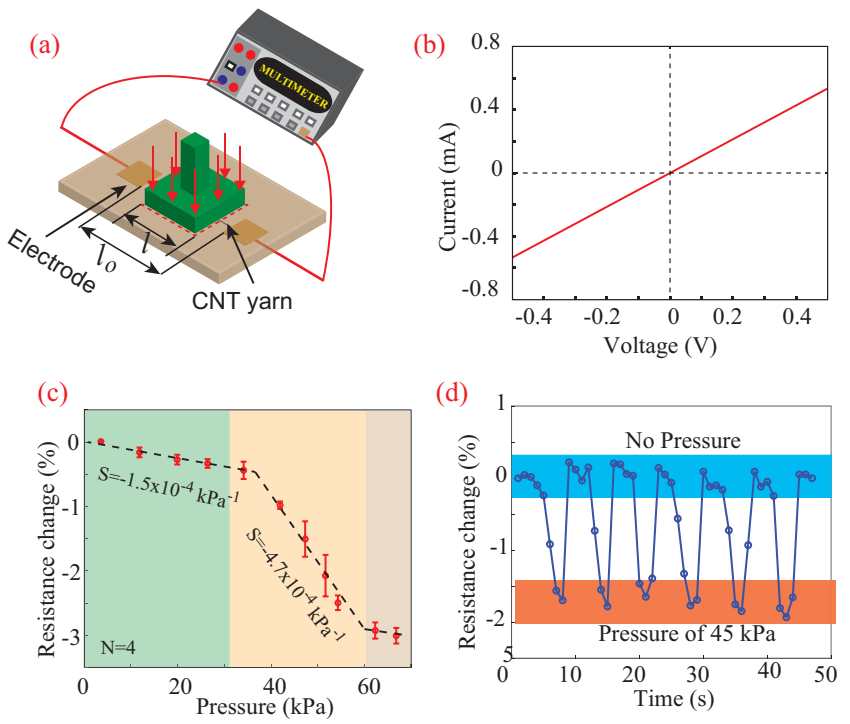

Fig. 2. Pressure effect on the electromechanical properties of CNT yarns. (a) Schematic sketch of experimental procedures with measurement method. (b) Current-Voltage characteristics of the as-fabricated yarns. (c) Relative resistance change of the yarn under pressure. Number of samples $\mathrm{N}=4$. (d) Repeatability of the electromechanical signal.

We embedded the yarn into a web of acrylic elastomer with a thickness of $500 \mu \mathrm{m}$ (i.e. 3M VHB Acrylic Foam Tape) (Figure 2a). The pressure test was performed using a force and displacement controlled machine (Instron 3367), while the resistance variation of the sensor was simultaneously monitored by LCR meter U1733C (Agilent/Keysight Technologies). The pressing area of the calibration probe was $2.5 \times 2.5 \mathrm{~cm}$ while the effective pressing area of the CNT yarn is determined by $A_{p}=l \times d$ where $l=2.5 \mathrm{~cm}$ is the effective length (under pressure) and $d$ is the diameter of the yarn. The total length of the yarn sample is $l_{o}\left(l_{o}>l\right.$ in order to avoid pressure applied to the electrode), the total resistance of the CNT yarn and the resistance of pressing portion are $R_{o}=\rho l_{o} / A$ and $R=\rho l / A$, respectively, where $\rho$ and $A=\pi d^{2} / 4$ are the resistivity and cross-sectional area of the yarn, respectively. Therefore, the resistance of the pressing portion can be calculated using the measured total resistance of the yarn as $R=R_{o} \times l / l_{o}$.

\section{RESULTS AND DISCUSSION}

Figure $2 b$ shows the linear current-voltage (I-V) characteristic of the CNT yarn, indicating the good Ohmic contact of the yarn and the electrodes. Figure $2 \mathrm{c}$ shows the resistance variation versus pressure response of the CNT yarn. Evidently, the electrical resistance of the CNT yarn decreases with increasing applied pressure (e.g. a maximum resistance decrease of $2.8 \%$ at an applied pressure of $60 \mathrm{kPa}$ ), indicating an improvement of its electrical conductivity. Accordingly, the pressure sensitivity (i.e. responsivity) of the CNT yarn $S=\Delta R / R \times 1 / P$, where $P$ is the applied pressure, was found to be $-1.5 \times 10^{-4}$ and $-4.7 \times 10^{-4} \mathrm{kPa}^{-1}$. This result is comparable to other highly sensitive pressure sensors with responsivity varying from $10^{-4}$ to $6.7 \times 10^{-4} \mathrm{kPa}^{-1}$ [15], [16], [17], [18]. In addition, the CNT yarn demonstrated excellent repeatability with several cycles of applied pressure (Figure $2 \mathrm{~d})$. The above desirable characteristics indicate the possibility of using CNT yarns for pressure sensing applications. Our study shows a resistance change of CNT yarns upto $2.8 \%$, while that reported in ref. [9] is less than 1\%. This is because the direct transverse pressure (this study) can significantly decrease the tunneling distance while tensile strain (ref. [9]) can slightly compact the yarn in the transverse direction [12]. The detailed mechanism of this phenomenon will be interpreted as follows.

The total resistance of the CNT yarn comes from the resistance of individual CNTs $\left(R_{C N T}\right)$ and their across junctions $\left(R_{\text {junction }}\right)$, where the contribution of the latter is dominant $\left(R_{\text {junction }}>>R_{C N T}\right)$ [12]. In other words, the CNT-CNT junction is the main barrier preventing the free-movement of electrons in the yarn under applied electric fields [19]. In addition, due to the weak Van der Waals interaction force between adjacent CNTs, although the applied pressure causes negligible effect on individual CNTs, it significantly increases the contact points of CNT yarns as well as reduces the tunneling distance. Therefore, the pressurising effect on the CNT junction is considered as a main contributing factor to the conduction of CNT yarns. Among the existing CNT junctions in the yarn, only junctions with effective tunneling distance (i.e. $\sim 1 \mathrm{~nm}$ ) play the important role in the CNT conductivity as the tunneling resistance exponentially depends on the tunneling distance $d$ between individual CNTs as follows [19]: 


$$
R_{\text {tunneling }}=\frac{h^{2} d}{A_{\text {cross }} e^{2} \sqrt{2 m \lambda}} \exp \left(\frac{4 \pi d}{h} \sqrt{2 m \lambda}\right)
$$

where $h, m$ and $e$ are the Plank's constant, the mass of electron and the electricity of electron, respectively; $A_{\text {across }}$ denotes the cross section of the tunnel; $\lambda$ is the height of the CNT-CNT barrier. It is believed that the number of CNT junctions with effective tunneling distance increases with increasing applied pressure (Figure 3a), and leads to an increase of the electrical path of electron; thus, the decrease in the resistance of the CNT yarn. However, due to the compactness of CNT yarns after a heat treatment, the increase of conducting paths is insignificant. This leads to a decrease of the resistance at a low pressure regime $(<30 \mathrm{kPa})$ with a relatively low sensitivity of $1.5 \times 10^{-4} \mathrm{kPa}^{-1}$ (Figure 2c). At a higher pressure regime (30$60 \mathrm{kPa}$ ), a small change of tunneling distance $\Delta d$ could result in a significant decrease of the electrical resistance (Figure $3 \mathrm{~b})$. The change of tunneling distance is associated with the change of the CNT volume fraction. As such, the volume fraction of CNT $V_{f o}$ before applied pressure is calculated as $V_{f o}=N V_{C N T} / V=N\left(\pi d_{C N T}^{2}\right) / 4 /\left(\pi D_{\text {yarn }}^{2}\right) / 4=$ $N\left(d_{C N T} / D_{\text {yarn }}\right)^{2}$, where $\mathrm{V}_{C N T}$ and $\mathrm{V}$ are the volume fraction of CNT and total volume of CNT yarn, respectively; $d_{C N T}$ and $D_{y a r n}$ are the diameters of individual CNT and CNT yarn, respectively; $\mathrm{N}$ is the number of individual CNTs counted in the cross-section of the yarn. The density function of CNT is calculated as $\Omega=1 /(\pi-\pi \cos \phi)$ [20], [21], where $\phi$ is the twisting angle at the surface of the yarn. Therefore, the number of CNT junctions (with minimum distance) per $\mu \mathrm{m}$ length is calculated as follows [20], [21]:

$$
n=\frac{8 V_{f o} I}{d\left(\pi+4 V_{f o} \Psi\right)}
$$

where $I=\frac{2 \phi-\sin 2 \phi}{4(1-\cos \phi)}$ and $\Psi=\frac{\phi(2 \phi-\sin 2 \phi)}{4(1-\cos \phi)^{2}}$. After the pressure is applied, the volume fraction is $V_{f}=V_{f o} D_{\text {yarn }}^{2} /(a b)$ where $\mathrm{a}$ and $\mathrm{b}$ are fundamental dimensions of the elliptic shown in Figure 3a. Comsol simulation was performed to qualitatively estimate the change of cross-section of the CNT yarn under pressure (not shown here). Simulation results indicate that

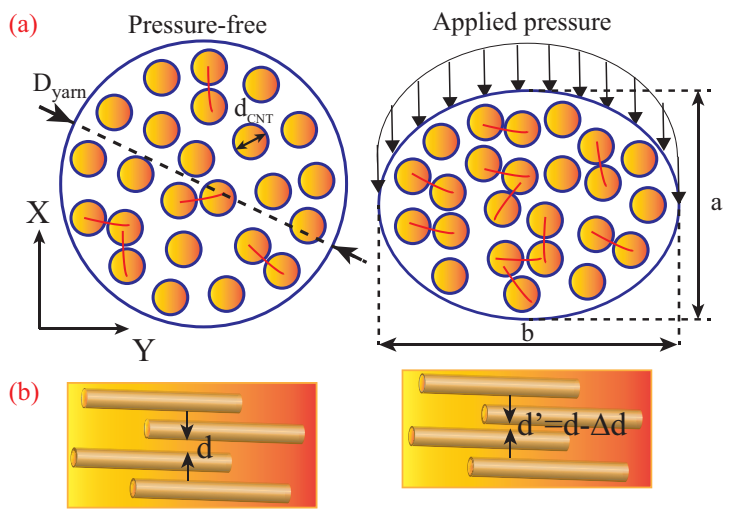

Fig. 3. Schematic sketch of the pressurising effect on the CNT junctions. (a) Pressurising effect increases the number of effective CNT junctions. (b) Pressurising effect on the tunneling distance between CNTs in CNT junctions. the total area of CNT cross-section decreases with increasing applied pressure, leading to an increase in the volume fraction of the CNT yarn $\left(V_{f}>V_{f o}\right)$. Therefore, it is evident from Eq. (2) that the number of CNT junctions increases with increasing $V_{f}$. As a result of applied pressure, the increase in CNT junctions can lead to the decrease of electrical resistance. We assume that the conductivity of the CNT yarn is proportional to the number of CNT junctions. In addition, the change of CNT volume fraction under pressure is expected to be small as the maximum resistance change is $2.8 \%$. Based on Eq (2), the relationship between resistance change and volume fraction change of CNT $\left(\Delta V_{f}=V_{f}-V_{f o}\right)$ is approximated as follows:

$$
\Delta R / R=-\alpha \Delta V_{f}
$$

where $\alpha$ is a constant. The increase of volume fraction of CNT leads to the highest sensitivity of $-4.7 \times 10^{-4} \mathrm{kPa}^{-1}$, as shown in Figure 2c. It is important to note that the electrical resistance is saturated because the tunneling distance reaches its minimum threshold of $60 \mathrm{kPa}$. The low pressure threshold achieved can be explained in terms of the stability of CNT volume fraction with an applied pressure of above $60 \mathrm{kPa}$ [22]. This is contributed to the high compactness of the well-aligned yarn using the heat treatment, and drafting and twisting system. Therefore, the CNT yarn is highly potential for pressure sensors working in the 0-60 kPa pressure range.

\section{CONCLUSION}

In conclusion, we investigated the impact of the radial pressure on the electromechanical properties of CNT yarns. A decrease in the electrical resistance of CNT yarns was observed under applied compressive pressure. The pressure sensitivity was found to be approximately $4.7 \times 10^{-4} \mathrm{kPa}^{-1}$ for the CNT yarns, which is favourable for pressure sensing applications. The compact and lightweight yarns were fabricated using heat treatment and aligned by the capstan rod system, showing a high potential for pressure sensing where the applications of pressure threshold lower than 60 $\mathrm{kPa}$ are applied. The integration of CNT yarns for mechanical sensors with a wider range of pressure detection will enable the practical application of CNT yarns for pressure sensing.

\section{ACKNOWLEDGMENT}

This work was performed in part at the Queensland node of the Australian National Fabrication Facility, a company established under the National Collaborative Research Infrastructure Strategy to provide nano and micro-fabrication facilities for Australia's researchers. This work has been partially supported by Australian Research Council grants LP150100153 and LP160101553. Toan Dinh acknowledges the support from 2017 Griffith Early Career Researcher Travel Grant.

\section{REFERENCES}

[1] P. M. Ajayan and O. Z. Zhou, "Applications of carbon nanotubes," in Carbon nanotubes, ed: Springer, 2001, pp. 391-425.

[2] M. Schulz, V. Shanov, and Z. Yin, Nanotube superfiber materials: changing engineering design: William Andrew, 2013. 
[3] R. H. Baughman, A. A. Zakhidov, and W. A. De Heer, "Carbon nanotubes-the route toward applications," science, vol. 297, pp. 787-792, Aug. 2002, doi: 10.1126/science.1060928.

[4] T. Dinh, H.-P. Phan, T.-K. Nguyen, A. Qamar, A. R. M. Foisal, T. N. Viet, C. D. Tran, Y. Zhu, N.-T.Nguyen, D. V. Dao, ”Environment-friendly carbon nanotube based flexible electronics for noninvasive and wearable healthcare," Journal of Materials Chemistry C, vol. 4, pp. 10061-10068, 2016, doi: 10.1039/C6TC02708C.

[5] C. Stampfer, A. Jungen, R. Linderman, D. Obergfell, S. Roth, and C. Hierold, "Nano-electromechanical displacement sensing based on singlewalled carbon nanotubes," Nano letters, vol. 6, pp. 1449-1453, Jul. 2006, doi: $10.1021 / \mathrm{n} 10606527$.

[6] V. T. Dau, T. Yamada, D. V. Dao, B. T. Tung, K. Hata, and S. Sugiyama, "Integrated CNTs thin film for MEMS mechanical sensors," Microelectronics Journal, vol. 41, pp. 860-864, Dec. 2010, doi: 10.1016/j.mejo.2010.07.012

[7] V. T. Dau, B. T. Tung, T. X. Dinh, D. V. Dao, T. Yamada, K. Hata, and S. Sugiyama, "A micromirror with CNTs hinge fabricated by the integration of CNTs film into a MEMS actuator," Journal of Micromechanics and Microengineering, vol. 23, p. 075024, Jun. 2013, doi: 10.1088/09601317/23/7/075024.

[8] V. T. Dau, D. V. Dao, T. Yamada, B. T. Tung, K. Hata, and S. Sugiyama, "Integration of SWNT film into MEMS for a micro-thermoelectric device," Smart Materials and structures, vol. 19, p. 075003, May. 2010, doi: 10.1088/0964-1726/19/7/075003.

[9] J. L. Abot, T. Alosh, and K. Belay, "Strain dependence of electrical resistance in carbon nanotube yarns," Carbon, vol. 70, pp. 95-102, Apr. 2014, doi: 10.1016/j.carbon.2013.12.077.

[10] W. Obitayo and T. Liu, "A review: carbon nanotube-based piezoresistive strain sensors," Journal of Sensors, vol. 2012, Apr. 2012, doi: $10.1155 / 2012 / 652438$.

[11] C.-D. Tran, W. Humphries, S. M. Smith, C. Huynh, and S. Lucas, "Improving the tensile strength of carbon nanotube spun yarns using a modified spinning process," Carbon, vol. 47, pp. 2662-2670, Sep. 2009, doi: 10.1016/j.carbon.2009.05.020.

[12] T.-K. Nguyen, T. Dinh, H.-P. Phan, C.-D. Tran, A. R. M. Foisal, Y. Zhu, D. V. Dao, "Electrically Stable Carbon Nanotube Yarn Under Tensile Strain," IEEE Electron Device Letters, vol. 38, pp.1331-1334, Sep. 2017, doi: 10.1109/LED.2017.2726016.

[13] C.-D. Tran, S. Lucas, D. Phillips, L. Randeniya, R. Baughman, and T. Tran-Cong, "Manufacturing polymer/carbon nanotube composite using a novel direct process," Nanotechnology, vol. 22, p. 145302, Feb. 2011, doi: 10.1088/0957-4484/22/14/145302.

[14] M. Miao, "Electrical conductivity of pure carbon nanotube yarns," Carbon, vol. 49, pp. 3755-3761, Oct. 2011, doi: 10.1016/j.carbon.2011.05.008.

[15] G. Darlinski, U. Bttger, R. Waser "Mechanical force sensors using organic thin-film transistors," Journal of applied physics, vol. 97, p. 093708, May. 2005, doi: 10.1063/1.1888046.

[16] C. Metzger, E. Fleisch, J. Meyer, M. Dansachmller, I. Graz, M. Kaltenbrunner, C. Keplinger, R. Schwodiauer, and S. Bauer, "Flexiblefoam-based capacitive sensor arrays for object detection at low cost," Applied Physics Letters, vol. 92, p. 013506, Jan. 2008, doi: $10.1063 / 1.2830815$

[17] D. J. Lipomi, M. Vosgueritchian, B. C. Tee, S. L. Hellstrom, J. A. Lee, C. H. Fox, Z. Bao, "Skin-like pressure and strain sensors based on transparent elastic films of carbon nanotubes," Nature nanotechnology, vol. 6, pp. 788-792, Dec. 2011, doi: 10.1038/nnano.2011.184.

[18] I. Graz, M. Krause, S. Bauer-Gogonea, S. Bauer, S. P. Lacour, B Ploss, M. Zirkl, B. Stadlober, S. and Wagner, "Flexible active-matrix cells with selectively poled bifunctional polymer-ceramic nanocomposite for pressure and temperature sensing skin," Journal of Applied Physics, vol. 106, p. 034503, Aug. 2009, doi: 10.1063/1.3191677.

[19] N. Hu, H. Fukunaga, S. Atobe, Y. Liu, and J. Li, "Piezoresistive strain sensors made from carbon nanotubes based polymer nanocomposites," Sensors, vol. 11, pp. 10691-10723, Nov. 2011, doi: 10.3390/s111110691.

[20] N. Pan, "A modified analysis of the microstructural characteristics of general fiber assemblies," Textile Research Journal, vol. 63, pp. 336-345, Jun. 1993, doi: 10.1177/004051759306300605.

[21] N. Pan, "Development of a constitutive theory for short fiber yarns: Mechanics of staple yarn without slippage effect," Textile research journal, vol. 62, pp. 749-765, Dec. 1992, doi: 10.1177/004051759206201208.

[22] S. V. Lomov, L. Gorbatikh, and I. Verpoest, "Compression behaviour of a fibre bundle with grafted carbon nanotubes," Carbon, vol. 49, pp. 4458-4465, Nov. 2011, doi: 10.1016/j.carbon.2011.06.044. 